\title{
Model Order Reduction in computational multiscale fracture mechanics
}

\author{
M. Caicedo ${ }^{1, a}$, J. Oliver ${ }^{1, b}$, A.E. Huespe ${ }^{2, c}$, and O. Lloberas-Valls $s^{1, d}$
}

${ }^{1}$ E.T.S. d'Enginyers de Camins, Canals i Ports, Technical University of Catalonia (BarcelonaTech)/International Center for Numerical Methods in Engineering (CIMNE) Campus Nord UPC, Edifici C-1, c/ Jordi Girona 1-3, 08034 Barcelona, Spain

${ }^{2}$ CIMEC-UNL-CONICET, Güemes 3450, Santa Fe, Argentina

amcaicedo@cimne.upc.edu, boliver@cimne.upc.edu, ${ }^{\mathrm{c}}$ ahuespe@intec.unl.edu.ar, dolloberas@cimne.upc.edu

Keywords: Model Order Reduction, Reduced-Order Cubature, Fracture, Computational Multi-scale modeling, Continuum Strong Discontinuituy Approach.

\begin{abstract}
Nowadays, the model order reduction techniques have become an intensive research field because of the increasing interest in the computational modeling of complex phenomena in multiphysic problems, and its consequent increment in high-computing demanding processes; it is well known that the availability of high-performance computing capacity is, in most of cases limited, therefore, the model order reduction becomes a novelty tool to overcome this paradigm, that represents an immediately challenge in our research community. In computational multiscale modeling for instance, in order to study the interaction between components, a different numerical model has to be solved in each scale, this feature increases radically the computational cost. We present a reduced model based on a multi-scale framework for numerical modeling of the structural failure of heterogeneous quasibrittle materials using the Strong Discontinuity Approach (CSD). The model is assessed by application to cementitious materials. The Proper Orthogonal Decomposition (POD) and the Reduced Order Integration Cubature are the proposed techniques to develop the reduced model, these two techniques work together to reduce both, the complexity and computational time of the high-fidelity model, in our case the $F E^{2}$ standard model.
\end{abstract}

\section{Introduction}

The present model departs from the multiscale framework developed in [2] for the numerical modeling of failure via hierarchical multi-scale models, taking advantage of the reduced order techniques developed in [1], the theoretical framework used in this work is based on the so-called $\left(F E^{2}\right)$ methods via first order computational homogenization for the coupling between scales, in which homogenized quantities at the lower scale, represented by a so-called failure-cell, are therefore transferred, in a one-way fashion, to material points (Gauss points) of the macroscopic structure. The formulation is presented in terms of strains in a non-conventional format imposing the natural multiscale boundary conditions via Lagrange multipliers. This work attempts to solve the problematic of excessive computational time in multi-scale models, in our case an additional complexity is induced by the discontinuous displacement field produced by the strain localization at both scales. Nonetheless, the methodology can also be straightforward extended to problems with continuous fields.

\section{Model description}

Generalities of $F E^{2}$ method applied to multiscale fracture problems: This approach is developed under a small strain framework, the equality of internal power at both scales is guaranteed via HillMandell Macro-Homogeneity principle. In this approach, the macroscopic constitutive response is proven to be point-wise equivalent to an inelastic law (in an incremental fashion) as a function of the homogenized elastic tangent tensor, $\mathbf{C}^{\text {hom }}$, and the incremental homogenized inelastic strain rate $\dot{\boldsymbol{\varepsilon}}^{(i)}$ i.e.: 


$$
\dot{\boldsymbol{\sigma}}=\mathbf{C}^{h o m}:\left(\dot{\boldsymbol{\varepsilon}}(x)-\dot{\boldsymbol{\varepsilon}}^{(i)}\right) \quad \dot{\boldsymbol{\varepsilon}}^{(i)}=\frac{1}{l_{\mu}}(\mathbf{n} \otimes \dot{\boldsymbol{\beta}})
$$

Where, the inelastic strain component $\dot{\boldsymbol{\varepsilon}}^{(i)}$ is expressed as a function of the homogenized variables taken from the lower scale, and represent the average value of the symmetrical tensor product between the strong discontinuity normal $\mathbf{n}$, and the rate of displacement jump $\dot{\boldsymbol{\beta}}$ of each cohesive band, belonging to the manifold of the mesoscopic failure mechanism $\mathcal{S}_{\mu}$, i.e. the mesoscopic crack. In addition, the so-called material characteristic length $l_{\mu}$ is defined as the ratio between the measure (volume or area) of the representative volume and the measure (surface or length) of the mesoscopic failure mechanism. The equations that govern the lower scales are the next:

Problem I: Given a macroscale strain $\varepsilon$, Find $\tilde{\boldsymbol{u}}_{\mu}$ such that $\varepsilon_{\mu}=\varepsilon+\nabla^{s} \tilde{\boldsymbol{u}}_{\mu}$ and:

$$
\int_{\mathcal{B}_{\mu}} \boldsymbol{\sigma}_{\mu}\left(\boldsymbol{\varepsilon}_{\mu}\right): \nabla^{s} \tilde{\boldsymbol{u}}_{\mu} d \mathcal{B}_{\mu}=0 \quad ; \forall \tilde{\boldsymbol{u}}_{\mu} \in \mathcal{V}_{\mu}^{u}:=\left\{\tilde{\boldsymbol{u}}_{\mu} \mid \int_{\mathcal{B}_{\mu}} \nabla^{s} \tilde{\boldsymbol{u}}_{\mu} d \mathcal{B}_{\mu}=\mathbf{0}\right\} ;
$$

Model Order Reduction techniques: The reduction process is divided into two sequential stages. The first stage consists of a common Galerkin projection, via Proper Orthogonal Decomposition POD for the meso-scale strain field, onto a small space (reduced-order space). For the second stage, the main goal is to reduce the number of integration points given by the standard Gauss quadrature, by defining a new scheme that efficiently determines optimal points and its corresponding weights so that the error in the integration of the reduced model is minimized (Reduced Order Cubature - ROC).

In order to provide the reduced model with the input parameters and entities, the general procedure is also divided into two parts, the first one (offline part) in which the projection operators for the mesoscale strain field and the parameters of the new integration cubature are computed. These data, together with the material and geometrical parameters, define the set of input parameters for the first and second stage (online part). By comparison with the standard $\left(F E^{2}\right)$ scheme, the proposed model in (2) can be redefined in term of strains in a generalized fashion, imposing the kinematic constraint (2-b) in an explicit way via Lagrange multipliers.

Problem IB: Given a macro-scale strain $\varepsilon$, find $\tilde{\varepsilon}_{\mu}$ and $\lambda$ satisfying:

$$
\left(\tilde{\varepsilon}_{\mu}\left(\varepsilon, d_{\mu}\right), \boldsymbol{\lambda}\left(\varepsilon, d_{\mu}\right)\right)=\arg \left\{\min _{\tilde{\varepsilon}_{\mu}} \max _{\boldsymbol{\lambda}} \Pi\left(\tilde{\varepsilon}_{\mu}, \boldsymbol{\lambda}\right)\right\} ; \quad \text { such that } \quad \dot{d}_{\mu}\left(\boldsymbol{y}, \boldsymbol{\varepsilon}_{\mu}\right)=g\left(\varepsilon_{\mu}, d_{\mu}\right)
$$

Where $\Pi$ is the homogenized potential of energy at the meso-scale.

Projection of strain field via POD: The reduction of the meso-scale strain field is based on the projection of the weak form of the discrete mechanical problem into a reduced manifold (reducedorder space), this reduced space is spanned by Ritz (globally supported) basis functions obtained via Singular Value Decomposition (SVD) of a set of snapshots taken from training tests computed during the offline part. Following this reasoning, the meso-scale strain fluctuation can be expressed as:

$$
\tilde{\boldsymbol{\varepsilon}}_{\mu}(\boldsymbol{y}, t)=\sum_{i=1}^{n_{\varepsilon}} \boldsymbol{\Phi}_{i}(\boldsymbol{y}) c_{i}(t)=\boldsymbol{\Phi}(\boldsymbol{y}) \boldsymbol{c}(t)
$$

Where $\boldsymbol{c}(t)=\left\{c_{1}, c_{2}, c_{3}, \ldots, c_{n_{\varepsilon}}\right\}$ is time dependent $\left(\boldsymbol{c} \in \mathbb{R}^{n_{\varepsilon}}\right)$ and represents the amplitude of the corresponding meso-scale strain mode updated during the online part. Now, introducing (4) into the Problem $I B$ and, after some straightforward manipulations, results into a new model written in terms of the reduced basis: 
Problem II: Given a macro-scale strain $\varepsilon$, find $c \in \mathbb{R}^{n_{\varepsilon}}$ satisfying:

$$
\int_{\mathcal{B}_{\mu}} \boldsymbol{\Phi}^{T}\left[\boldsymbol{\sigma}_{\mu}(\boldsymbol{\varepsilon}+\boldsymbol{\Phi} \boldsymbol{c})+\boldsymbol{\lambda}\right] d \mathcal{B}_{\mu}=\mathbf{0} ; \quad \text { tal que } \quad \int_{\mathcal{B}_{\mu}} \boldsymbol{\Phi}(\boldsymbol{y}) \boldsymbol{c}(t) d \mathcal{B}_{\mu}=\mathbf{0} ;
$$

Solving the system of equations (5) for $c$ and $\boldsymbol{\lambda}$ (Lagrange multiplier to ensure the equality of internal power at both scales via Hill-Mandel Macro-Homogeneity principle), it can be immediately noticed that this problem with $n_{\varepsilon}+n_{\sigma}$ equations will be cheaper, (in computational cost terms), than the standard $\left(F E^{2}\right)$ framework. However, the matricial form of Problem II has to be computed (in a standard way) prior its projection onto the reduced-order space. This fact highlights that the actual bottleneck for fast online computation is not the solution of the discrete balance equations but, rather, the determination of the stresses. To persue the main objective of the second stage, we develop a Hyperreduced Order Model (HPROM) via Reduced Optimized Cubature (ROC), this technique is based on a discrete minimization problem that allows determining the optimized location of integration points and the corresponding weights.

The success of our proposed scheme, relies on the fact that it is possible to find a set of integration points $n_{r}$, substantially smaller than the ones given by the Gauss standard quadrature, minimizing the error in the assessment of (5). Introducing the new integraton rule into the Problem II, we get:

Problem III: Given the macro-scale strain $\varepsilon$, find $c \in \mathbb{R}^{n_{\varepsilon}}$ satisfying:

$$
\sum_{j=1}^{n_{r}}\left(\boldsymbol{\Phi}\left(\boldsymbol{z}_{j}\right)^{T} \boldsymbol{\sigma}_{\mu}\left(\boldsymbol{z}_{j}, \boldsymbol{c}\right)\right) \omega_{j}+\int_{\mathcal{B}_{\mu}} \boldsymbol{\Phi}^{T} \boldsymbol{\lambda} d \mathcal{B}_{\mu}=0 ; \quad \text { tal que } \int_{\mathcal{B}_{\mu}} \boldsymbol{\Phi}(\boldsymbol{y}) \boldsymbol{c}(t) d \mathcal{B}_{\mu}=\mathbf{0}
$$

\section{Numerical Results: Application to simulation of fracture in cementitious materials}

The macro-scale will be splitted into two subdomains, the dark gray domain will be modeled using an elastic monoscale constitutive law, taking the elastic homogenized constitutive tangent tensor, and, in the green domain the Hiper-Reduced Order Model (HPROM). The finite element mesh of the mesoscale is also depicted in figure (1-b), Material properties have been taken from [3].

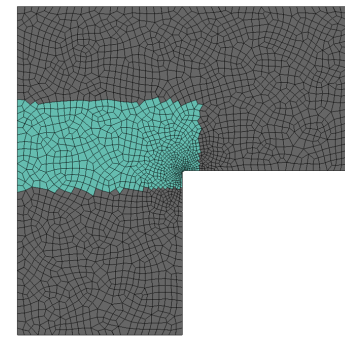

(a) Macroscale FE discretization

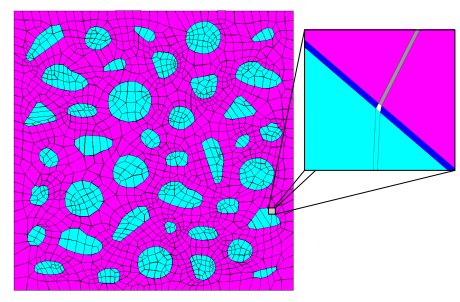

(b) Meso-scale FE discretization

\begin{tabular}{cccc} 
Mortar Matrix & & & \\
\hline Young modulus & $E_{m}$ & {$[\mathrm{MPa}]$} & 18500 \\
Poisson's ratio & $v_{m}$ & {$[-]$} & 0.18 \\
Tensile strength & $f_{t, m}$ & {$[\mathrm{MPa}]$} & 2.60 \\
Fracture energy & $G_{f, m}$ & {$[\mathrm{~N} / \mathrm{m}]$} & 140 \\
Aggregates & & & \\
\hline Young modulus & $E_{a}$ & {$[\mathrm{MPa}]$} & 37000 \\
Poisson's ratio & $v_{a}$ & {$[-]$} & 0.18 \\
(Unger \& Eckard 2011) & &
\end{tabular}

(c) Material properties

Fig. 1: Finite element discretization and material properties

The figure (2-a) shows the structural response in terms of load-displacement $(\mathbf{P}-\delta)$ curve (vertical load of the bottom, rightmost corner node versus displacement at the same place) for each set of strain modes $n_{\varepsilon}$ and integration points $n_{r}$. It is also shown the sensitivity in the convergence of the structural behavior as $n_{\varepsilon}$ increases. In figure (2-b), it can be observed the convergence results for the meso-scale 
tests using the Hiper-Reduced Order Model; fixing a number of strain modes $n_{\varepsilon}$, we get an optimal number of integration points. In addition, it can be immediately noticed that, as the number of strain modes $n_{\varepsilon}$ increases, the error decreases monotonically. The imposition of a judicious equilibrium between error and number of integration points plays an important role in the good performance of the method.

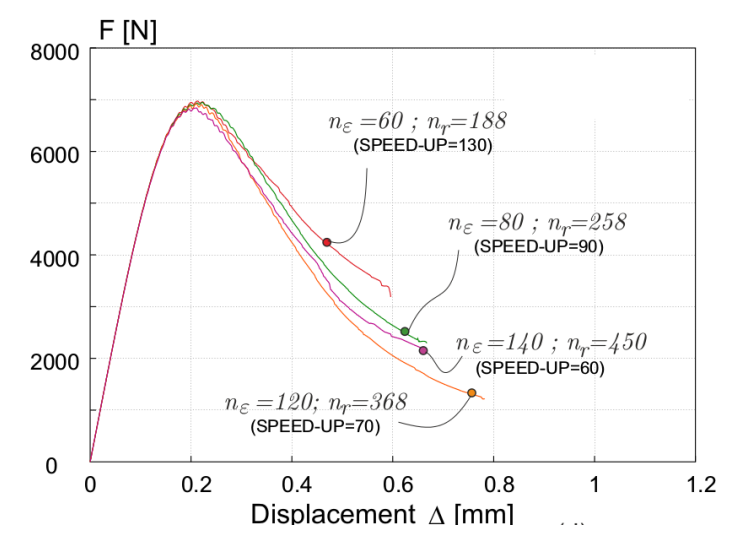

(a) Macro-structural response - L Shape Panel
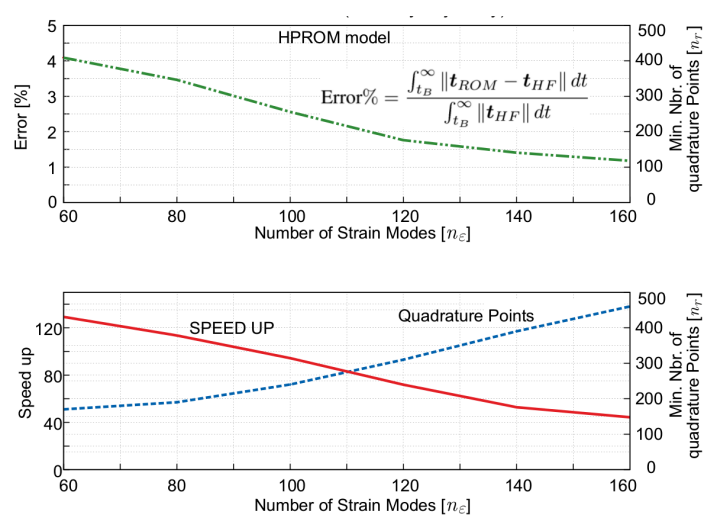

(b) Convergence analysis of the meso-scale

Fig. 2: Convergence error in macro and meso scales

\section{Conclusions}

The result of this work is a reduced model based on a hierarchical $\left(F E^{2}\right)$ multiscale approach for material failure in cementitious materials, that preserves all features of the standard FE model [2]. Furthermore, the two presented simulations show the convergence of the meso-scale and the sensitivity of the macro-structural behavior, as a function of the amount of strain modes, $n_{\varepsilon}$, and the number of integration points, $n_{r}$. The reduced model solves the problem of unafordable computational cost.

\section{Acknowledgments}

The research leading to these results has received funding from, on the one hand, the European Research Council under the European Union's Seventh Framework Program (FP/2007-2013) / ERC Grant Agreement n. 320815, Advanced Grant Project COMP-DES-MAT, and, on the other hand, the Spanish Ministry of Economy and Competitiveness through the National Research Plan 2014: MAT201460919-R. Oriol Lloberas-Valls gratefully acknowledges the funding received from the Spanish Ministry of Economy and Competitiveness through the "Juan de la Cierva" Postdoctoral Junior Grant: JCI-2012-13782 and the National Research Plan 2014: MAT2014-60919-R.

\section{References}

[1] Hernandez, J.A. and Oliver, J. and Huespe, A.E. and Caicedo, M.A. and Cante, J.C.: Highperformance model reduction techniques in computational multiscale homogenization(Computer Methods in Applied Mechanics and Engineering, Vol. 276, p 149--189, 2014).

[2] J. Oliver, M. Caicedo, E. Roubin, A. E. Huespe, and J.A. Hernandez: Continuum approach to computational multi-scale modeling of propagating material failure. Computer Methods in Applied Mechanics and Engineering, 294: 384-427, $2015 .$.

[3] J. F. Unger and S. Eckardt: Multiscale modeling of concrete - from mesoscale to macroscale. Archives of Computational Methods in Engineering, 18:341-393, 2011. 